

FIGURE

\section{Understanding anaesthesia}

To the Editor:

With financial support from ICI Pharma, the Foothills Hospital produced a 20-min patient education video in 1989 entitled "Understanding Anaesthesia" which was shown at the Canadian Anaesthetists' Society annual meeting in 1989. We were interested in studying the acceptability of this video to patients and the uses it would find in our hospital. Initially, it was shown only on the hospital's closed circuit television network and was seen by patients without specific direction. Because of interest shown by nurses, the video was soon being used for patient education classes. With the aid of a patient education specialist, we designed a questionnaire which studied the usefulness and acceptability of this video to these patients. In the initial survey, 27 questionnaires were completed. Most patients had had previous anaesthetic experience: $76 \%$ of patients rated the video as very good or excellent. Over $80 \%$ of respondents felt the program to be helpful, of the right duration, and understandable.

The video consisted of three parts: an introductory dramatic scenario depicting adminstration of the first anaesthetic in Canada; a short cartoon showing the historical milestones in the development of anaesthesia; and a dramatization of the tasks performed by the anaesthetist in patient care. The questionnaire showed that the cartoon segment was received negatively by about $30 \%$ of patients. It was subsequently removed from the presentation made to this group. In a follow-up survey of 154 patients, $97 \%$ of patients rated the modified video as very good or excel- lent. In another survey, the original video was shown to 21 postoperative patients who were given a similar questionnaire. Again, the video was well received but less so than by preoperative patients. All of these patients felt that the video would have been useful if seen preoperatively, Finally, the third segment of the video has been used in the training staff of nurses, although it has not been assessed in that role.

Although little has been reported about patient education in anaesthesia, this activity has potential benefits. For example, education might produce lower levels of preoperative anxiety. By creating more informed "consumers," it may provide a form of medicolegal "Risk Management." We hope to look at these questions in future. At present, it appears that information derived from questionnaires can aid the design of patient education materials.

\section{C.J. Eagle MD FRCPC \\ Department of Anaesthesia \\ University of Calgary}

\section{But yet, I'll make assurance double sure}

\section{To the Editor:}

Disconnection of the patient from the anaesthetic breathing system persists as an unpredictable, yet potentially catastrophic, event in the life of the patient and anaesthetist. ${ }^{2}$

Despite remarkable advances in patient and equipment monitoring and national and international recommendations for their use, we suspect that the frequency of disconnections has not decreased. In our own practice we find the multiplicity of connections, especially at the patient end of the system, to be a constant source of anxiety. This is particularly so when the airway is not directly and immediately available for inspection, as in head and neck surgery or when the patient is prone. During such cases we often revert to manual ventilation to ensure instantaneous detection of disconnection and to the use of adhesive tape to reinforce any "doubtful" connections. However, manual ventilation is not without hazard and inconvenience, and "the fixing of catheter mount connections with adhesive tape" has been cited recently as a factor hindering the resolution of negative events in anaesthesia. ${ }^{3}$ Personal experience of reconnecting previously taped but failed connections supports this view. Therefore, although we are unlikely ever to trust completely any connector, we believe that change is in order.

The pen with which this letter was drafted is a disposable plastic device with a push-on/pull-off cap. When the 